\title{
Finding of the mechanical power distribution in a horizontal ring mill using infrared thermography.
}

\author{
by G. Zannis*, M. Founti*, P. Makris*
}

Laboratory of Heterogeneous Mixtures and Combustion Systems, School of Mechanical Engineering - National Technical University of Athens, Greece

\begin{abstract}
The ring mil is a high energy-intensity machine used here for the pulverization of olivine with top size $750 \mu \mathrm{m}$. Infrared thermography is employed for the improvement of the pulverization efficiency as well as for improvement of the characteristics of the process along the mill. The material is ground on the inner mill surface and the work of the friction forces is converted into heat. The temperature of the mill surface during the startup is recorded as part of the experimental procedure. Results show that the temperature field is not uniform and the area of maximum heat generation lies close to the material entrance in the mill. Taking into account the above results the milling procedure can be altered for more efficient production.
\end{abstract}

\section{Introduction}

In general, comminution is the process of breaking particles into smaller ones and includes 3 stages which are distinguished mainly from the size of the initial material and the final product: a) crushing which refers to breaking rocks with dimension that range from meters to several centimeters and is accomplished by crushers, b) grinding (or milling) which uses mills and produces material with size from millimeters to micrometers and c) pulverization which uses mill-pulverizers for the production of fine material with maximum size several decades of micrometers ( $\mu \mathrm{m}$ where $1 \mu \mathrm{m}=10^{-3} \mathrm{~mm}$ ). The limits between the 3 stages are usually overlapped. Comminution is an inevitable unit operation in mineral processing and also by far the most energy consuming process in the mineral plants accounting for $90 \%$ of the energy required in ore processing [1,2].

The products of the comminution, especially the fine powders, find application either as final products or in the production of a wide spectrum of industrial and consumer products, such as pharmaceuticals, paper, paints, plastics, adhesives, construction products, composite materials etc.

In the relevant literature [2] comminution efficiency does not exceeds $5 \sim 10 \%$, with most researchers reporting efficiencies around 1\% (defined as the fraction of the mechanical energy given to the mill that is converted to new surface energy of the particles). In general, the energy supplied to a mill is converted almost entirely to thermal energy, resulting in the increase of the temperature of the working parts of the mill e.g. the rings in the ring mill, the balls in the ball-mill, the rod in the rod-mill etc as well as the temperature of the produced material. It becomes obvious that new experimental approaches are needed to allow assessment of the energy consumption and thus improve comminution efficiency.

The aim of present work is the identification of regions with intense heat release in order to support the understanding and eventual improvement of the comminution process along a horizontal ring mill (Figure 1). Observation of the internal fast rotating parts of the mill during operation and measurement of the local temperature 


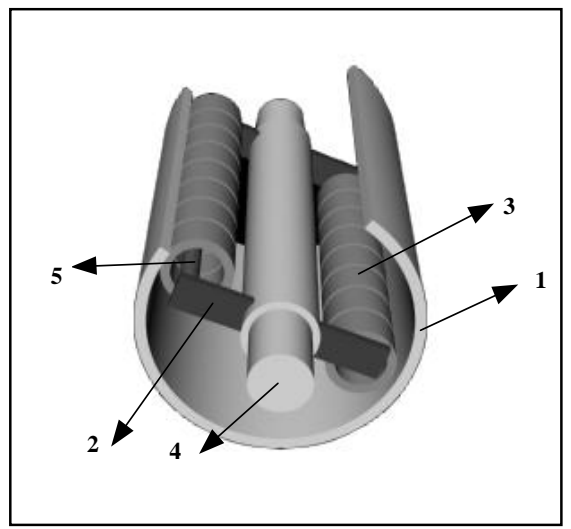

Fig. 1. Lay-out of the ring-mill

with conventional methods (e.g. thermocouples) is impossible due to lack of direct access. To overcome the above hurdles, the temperature distribution on the mill outer surface was measured with the aid of an infrared camera (FLIR PM595) and post processing of the thermographs took place with appropriate software. Experimental work done in the past with the currently used ring-mill has shown that its efficiency is approximately $5 \%[3,4]$.

\section{Experimental set -up and measuring procedure}

Figure 1 shows the main components of the ring mill that has been developed in NTUA. A cylinder (1) forms the main body of the mill. A rectangular metal frame is positioned along the principal axis of the cylinder and is attached to the main shaft (4). The main shaft can be rotated with the help of an electric motor. The rectangular metal frame comprises two arms (2) that are held parallel with the help of two ringconnecting rods (5). A variable number of annular metal rings (3) are inserted along each connecting rod. Each ring is allowed to move freely along and around each rod. During rotation of the orthogonal metal frame, the metal rings are compressed against the inner surface of the cylinder due to centrifugal forces. Simultaneously, frictional forces are developed between the metal rings and the inner surface of the machine. Thus, the rings rotate freely around their symmetry axis and simultaneously slide along the arms of the frame and roll on the inner surface of the cylinder. The particles are ground in the space left between the inner surface of the mill and the annular metal rings. The material is constantly swept by the rings and trapped between the rings and the inner surface of the mill. Consequently, particles are under the effect of normal (compaction), $\mathrm{P}$, and shear forces, $\mathrm{F}$ (Figure 2).

The mill diameter, $D_{c y l}$, is equal to $210 \mathrm{~mm}$ and the active ring length, namely the total length of the rings without gap among them, $L_{r}$, is $320 \mathrm{~mm}$. The active surface of the mill, which is the surface swept by the rings, $\mathrm{S}_{\text {act }}=\pi \cdot D_{c y l} \cdot L_{r}$, is calculated equal to $0.21 \mathrm{~m}^{2}$ and the active volume of the cylinder which corresponds to the active length, $\mathrm{V}_{\mathrm{act}}=\frac{\pi \cdot D_{c y l}^{2}}{4} L_{r}$, is equal to 11 lit. 


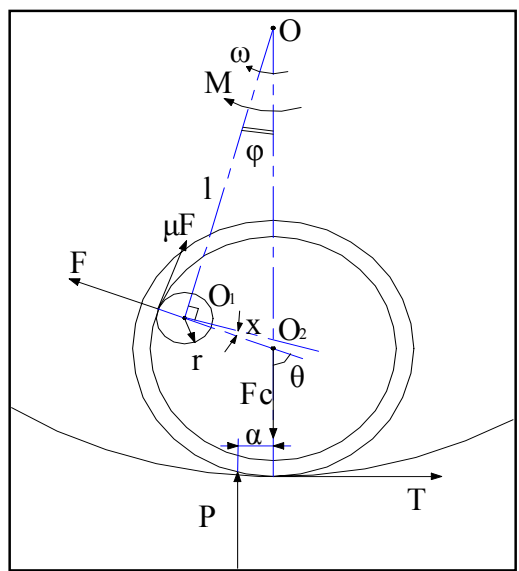

Fig. 2. Forces exerted on the ring

The operation of the machine has proven to be highly energy intensive. The material ground is olivine - Magnesium Iron Silicate $(\mathrm{Mg}, \mathrm{Fe})_{2} \mathrm{SiO}_{4}$ - that has a high value of hardness, corresponding to ca. 7 on the Mosh hardness scale. The mass throughput is $30 \mathrm{~kg} / \mathrm{h}$. The maximum dimension of the particle feed size (97\%) at the inlet of the mill is $-0.750 \mathrm{~mm}$ while the achieved final size is $-0.075 \mathrm{~mm}$. The mill operating parameters - rotational speed and raw material throughput - are kept constant during the experiment. The initial temperature of the mill surface is $30^{\circ} \mathrm{C}$ while the final recorded is $62^{\circ} \mathrm{C}$. The duration of the experiment is $270 \mathrm{~s}$.

During experiments the mill is operated with the aid of an electric motor. A torque sensor set between the mill and the motor is used to measure the mechanical power absorbed by the mill, found equal to $1800 \mathrm{~W}$. The power losses due to the bearings are excluded. From the above the specific powers are derived as:

$$
\begin{aligned}
& \text { Surface power } P_{\text {surf }}=\frac{P}{S_{a c t}}=8.57 \mathrm{~kW} / \mathrm{m}^{2} \\
& \text { Volumetric power } P_{v o l}=\frac{P}{V_{a c t}}=164 \mathrm{~kW} / \mathrm{m}^{3}
\end{aligned}
$$

It should be noted that the expected power of a conventional ball mill of similar dimensions is approximately $50 \mathrm{~W}$, which is 36 times smaller than the power of the ring mill. The mechanical energy supplied to the mill is mainly dissipated as heat increasing the temperature of the mill (cylinder and rings) and of the material. A small portion is converted into new surface energy, which is the energy used for the fragmentation of the particles. The rest of the energy is converted to noise and miscellaneous losses, like heating of the air inside the mill. The heat from the mill surface is transferred to the environment mainly through radiation and to a smaller extent through convection. In the present work only the radiated heat is considered.

The temperature distribution on the cylinder surface is measured with an infrared camera, model FLIR PM 595. The distance between the camera and the mill is $2 \mathrm{~m}$. The visual axis of the camera intersects the axis of the mill (Figure 3 ) minimizing the errors of the camera readings due to the inclination of the surface relatively to the 
axis of vision of the camera. The external surface of the mill is covered homogeneously with paint ensuring the same emissivity factor along the surface. The vertical sidewalls of the mill are in contact with the cylindrical surface and local heat transfer phenomena are expected.

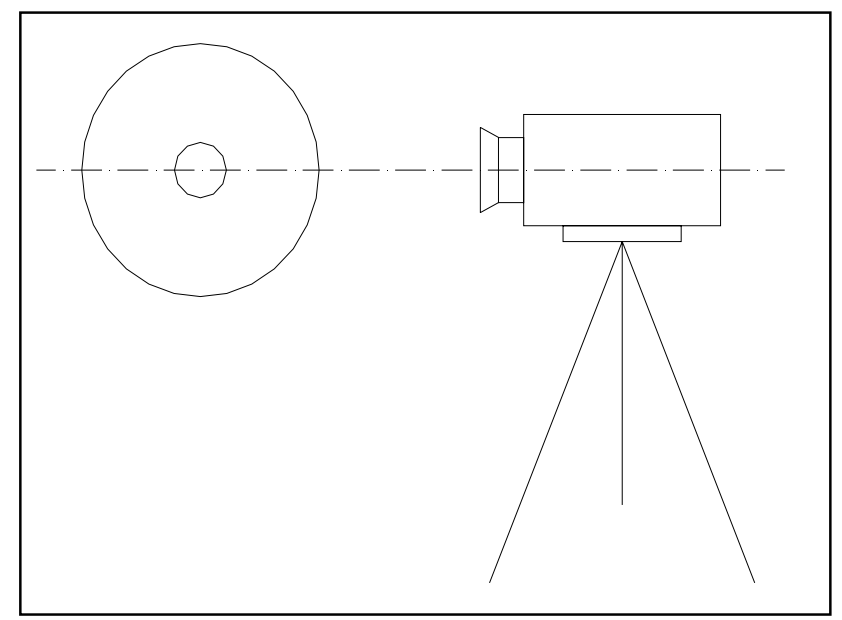

Fig. 3. Position of the camera in relation to the mill axis

The measurement accuracy of the IR-camera is $\pm 2 \%$ and its thermal sensitivity is less than $0.1 \mathrm{~K}$. Its detector consists of $320 \times 240$ pixels, while its spectral range is 7.5-13 $\mu \mathrm{m}$. The sampling rate of the camera in the present configuration has been approximately 2 thermograms/sec. The thermal data have been processed using the commercial software "ThermaCAM Researcher 2001", which estimates the surface temperature of the object in view, taking into consideration the emissivity of the object, the distance between the object and the camera and the conditions of the ambient environment (temperature, relative humidity). The images (thermographs) have been post-processed using a PC.

Sources of measurement inaccuracy can be associated with background radiation from the hot surface of the steel enclosure and with the estimation/variation of the emissivity factor. The thermocamera was placed about $30 \mathrm{~cm}$ away from the cylinder surface, pointing towards the surface at an appropriate angle in order to avoid Narcissus effects. The emissivity factor was determined on the basis of comparative measurements with thermocouples that were placed on the surface of the mill.

\section{Results}

A representative thermograph of the mill external surface after $60 \mathrm{~s}$ of operation is shown in Figure 4. The temperature line along which the temperature profiles of Figure 5 are extracted is indicated. The temperature line is a feature of the software accompanying the camera. The software extracts the temperature of every pixel of the line on the thermogram. The direction of flow of the material inside the mill is from right to left of both Figure 4 and Figure 5. 
http://dx.doi.org/10.21611/qirt.2006.029

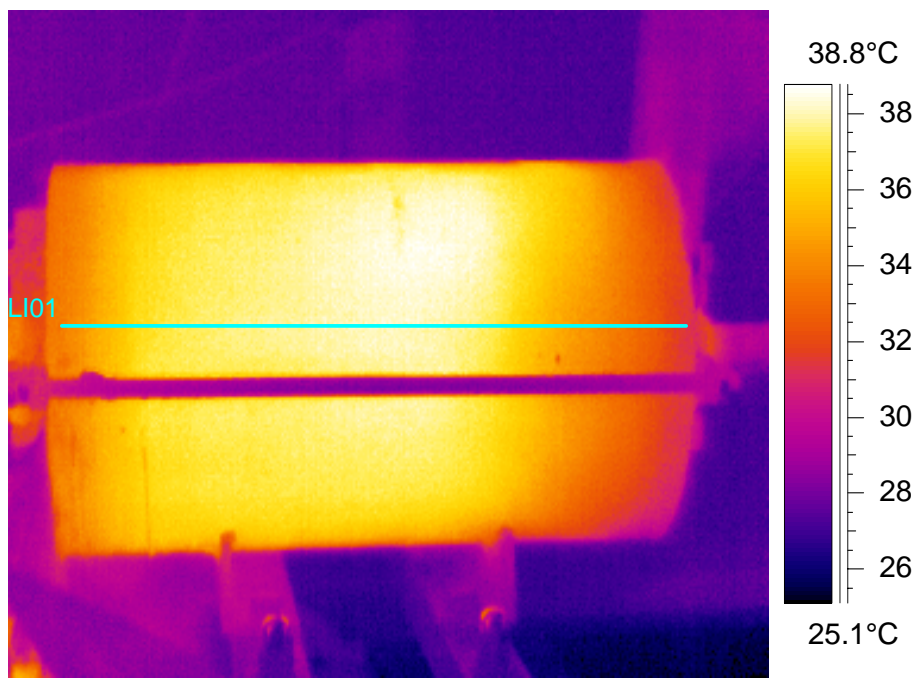

Fig. 4. Thermograph of the mill with the temperature line L01

The temperature profiles of Figure 5 are extracted from the corresponding thermographs that have been recorded in temperature intervals of $30 \mathrm{sec}$. They indicate that heat release from the mill outer surface is not uniform. Maximum temperatures, highlighting the location of maximum heat release, are measured at positions corresponding to ca. 1/3 of the distance from the entrance of the material. In fact, the location of maximum energy yield from the surface was expected to be close to the mill outlet. Misalignment of the shaft and of the mill rods relatively to the main enclosure has been excluded as a possible reason for the observed temperature distribution, after inspection of the mill alignment, which confirmed that the geometrical tolerances were within the expected limits.

Convection effects, at the two vertical sidewalls, result to lower temperature at the edges of the mill, as shown in Figure 5. Furthermore, relatively lower temperatures are measured at the mill inlet, since the material enters at ambient temperature, cooling locally the mill. Careful examination of Figure 5 indicates that the location of the maximum temperature measured in each profile (indicated in the Figure by a cross) moves in the early stages of the experiment towards the mill inlet and later on towards the outlet. This is associated with convection effects in the mass of the tube.

If the energy release along the mill had been uniform, the temperature would have increased in an exponential form, from the inlet to the outlet of the mill, taking into account the heat transfer mechanisms between the material and the mill during the warming up of the material and of the mill. The obtained results of Figures 4 and 5 suggest that the phenomenon depends on the grinding mechanism inside the mill.

It can be anticipated that the initially thick feed-material resists to the motion of the rings causing the maximum heat generation. The fine material acts as lubricant between the rings and the mill. According to [5] the fine material forms an elastic film absorbing the forces from the mill media and "protecting" the larger particles reducing 


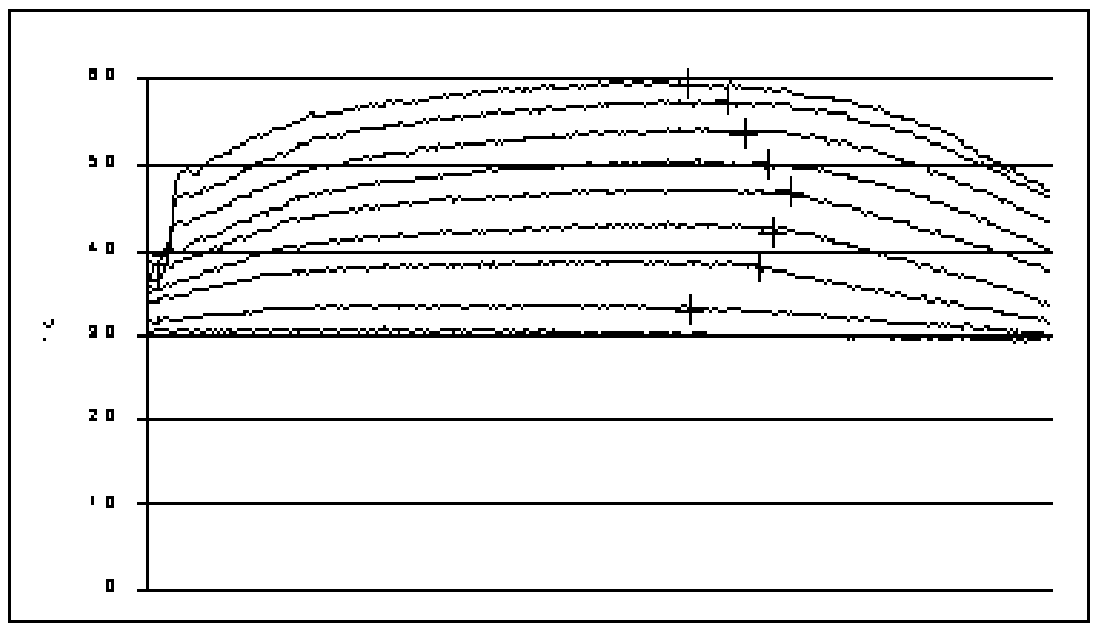

Fig. 5. Temperature distribution along the mill in relation with time.

the rate of particle breakage. This is one of the reasons that the fine material should be removed from the mill during operation.

The present configuration completes most of the grinding before the material reaches the middle of the mill. This doesn't mean that the material is not any more ground, but that the particle breakage rate is decreased. From a design optimization point of view, it can be recommended to reduce the effective length of the mill to approximately half of its current value. A second ring-mill, adapted to the fine material, can be serially connected to complete the pulverisation. The adaptation can be accomplished by altering the dimensions of the mill and the feed rate of the material, thus increasing the efficiency of the grinding process. However, it should be noticed that it's not the efficiency of the comminution itself that will be increased, but the efficiency of the grinding process, reducing the total amount electric energy necessary for the production of the same product. The above results can be taken into account for the dimensioning of the mill for specific production requirements and for the design of appropriate cooling system.

\section{Conclusions}

Infrared thermography is employed for investigation and comprehension of grinding mechanism in a horizontal ring mill by examination of the temperature field on the mill's external surface. It has been unveiled that the temperature field is not as theoretically expected along the mill. The maximum temperature was recorded near the entrance of the mill, implying that, under the present conditions, grinding is more intensive -and efficient- at that area. Using the above findings as a base, the design of the mill but also the design of the whole process can be altered for the improvement of the process efficiency. Overall, it has been shown that IR thermography can be a used as a reliable and flexible tool, capable of depicting temperature variations in energy intensive machine operation and can directly support design optimization. 


\section{REFERENCES}

[1] S. Alvorado et al, Energy-exergy optimization of comminution, Energy 23, No2 (1998) 153-158.

[2] S.S. Narayanan Modelling the performance of industrial ball mills using single particle breakage data Int. J. Mineral Processing 20 (1987) 211-228.

[3] G. Zannis, M. Founti, P. Makris Estimation of the grinding efficiency of a horizontal ring mill with the use of IR-thermography 6th Intern. Conference on Quantitative Infrared Thermography, QIRT 2002 Dubrovnik Croatia, (2002).

[4] M. Founti, G. Zannis, E. Tsolis, P. Makris Influence of design parameters on the energy balance of a horizontal ring mill Engineering Design Conference 2002 London (2002).

[5] B. Beke Principles of comminution House of the Hungarian Academy of Sciences (1964). 